\title{
ON NORMALISER PRESERVING LATTICE ISOMORPHISMS BETWEEN NILPOTENT GROUPS
}

\author{
D. W. BARNES and G. E. WALL
}

(received 10 May 1964)

\section{Introduction}

Let $\mathscr{L}(G)$ denote the lattice of all subgroups of a group $G$. By an $\mathscr{L}$-isomorphism (lattice isomorphism) of $G$ onto a group $H$, we mean an isomorphism of $\mathscr{L}(G)$ onto $\mathscr{L}(H)$. By an $\mathscr{N} \mathscr{L}$-isomorphism (normaliser preserving $\mathscr{L}$-isomorphism) of $G$ onto $H$, we mean an $\mathscr{L}$-isomorphism $\phi$ such that $\mathscr{N}\left(A^{\phi}\right)=\mathscr{N}(A)^{\phi}$ for all $A \in \mathscr{L}(G)$. In this paper, we study certain properties of groups which remain invariant under $\mathscr{N} \mathscr{L}$-isomorphisms.

In $\S 1$, we show that $\mathscr{N} \mathscr{L}$-isomorphisms can be characterised both as commutator preserving $\mathscr{L}$-isomorphisms and as mixed commutator preserving $\mathscr{L}$-isomorphisms. This result is closely related to recent work of Spring [11] on $\mathscr{L}$-isomorphisms between finite $p$-groups of exponent $p$. Spring proved, amongst other things, that every $\mathscr{L}$-isomorphism between such groups is an $\mathscr{N} \mathscr{L}$-isomorphism and that $\mathscr{L}$-isomorphic $k$-generator $p$-groups of exponent $p$ and class 2 are necessarily isomorphic if $k \leqq 4$. We give a simple derivation of Spring's first result and of an analogous one of Pekelis [7] for locally nilpotent, torsion-free groups.

Rottländer [9] has given an example of an $\mathscr{N} \mathscr{L}$-isomorphism between non-isomorphic finite groups of the same order. In $\S 2$, we give examples of the same phenomenon between finite $p$-groups ${ }^{1}(p>2)$. The groups in the simplest examples are of order $p^{4}$, exponent $p^{2}$ and class $3(p>3)$, and there are somewhat more complicated examples in which the groups have exponent $p(p>5)$.

Theorem 2 (§ 4) deals with the effect of an $\mathscr{N} \mathscr{L}$-isomorphism $\phi: \mathscr{L}(G) \rightarrow \mathscr{L}(H)$ on the central sections of $G$. (A section of $G$ means a factor group $A / B$, where $B \unlhd A \leqq G$. The section is called central if $B \unlhd G$ and $A / B \leqq \mathscr{Z}(G / B)$.) A typical case of the theorem states that if the restriction of $\phi$ to the factor commutator group $G / G^{\prime}$ is induced by an

1 It may be mentioned that the well known $\mathscr{L}$-isomorphisms between non-isomorphic modular $p$-groups are not $\mathscr{N} \mathscr{L}$-isomorphisms. Indeed, it can be deduced from Iwasawa's

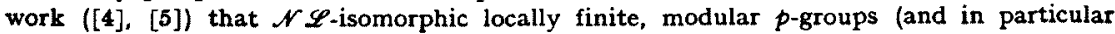
$\mathscr{L}$-isomorphic abelian $p$-groups) are necessarily isomorphic. 
isomorphism, then the same is true for the restriction of $\phi$ to every factor group $G_{(i)} / G_{(i+1)}$ of the descending central series. The proof uses the calculus of commutators and a general lemma (lemma 2, § 3) about multilinear mappings. This lemma is a wide generalisation of the fact that bilinear forms $f(x, y), g(x, y)$ which vanish for the same pairs of vectors $x, y$ are scalar multiples of one another.

Theorem 2 is applied to prove that, in certain cases, $\mathscr{N} \mathscr{L}$-isomorphic groups must be isomorphic. Theorem 3 affirms ${ }^{2}$ that this is so when the groups belong to a nilpotent variety $V$ and one of them is an almost free group of $V$. Here, an almost free group of $V$ means a factor group $F / M$, where $F$ is a free group of $V$ and $M$ is properly contained in the penultimate member of the descending central series of $F$.

A consequence of theorem 3 is that (contrary to Spring's ${ }^{3}$ assertion) $\mathscr{L}$-isomorphic $k$-generator $p$-groups of exponent $p$ and class 2 are necessarily isomorphic, whatever the value of $k$. The examples cited above show that this result does not generalise to groups of exponent $p$ and class $>2$.

Because of the analogy between commutators in nilpotent groups and products in nilpotent Lie algebras", analogous theorems about lattice isomorphisms between nilpotent Lie algebras are to be expected. Let $\mathscr{F}$ be a commutative ring with 1 and $L$ a Lie algebra over $\mathscr{F}$. We denote by $\mathscr{L}(L)$ the lattice of subalgebras of $L$. By an $\mathscr{L}$-isomorphism of $L$ onto a Lie algebra $M$ over the same ring $\mathscr{F}$, we mean an isomorphism of $\mathscr{L}(L)$ onto $\mathscr{L}(M)$. We shall merely state the principal results for Lie algebras during the course of the paper, indicating points at which the proofs differ significantly from those for groups.

Notation. Let $x, y, x_{1}, x_{2}, \cdots$ be elements of a group $G .\left\langle x_{1}, x_{2}, \cdots\right\rangle$ denotes the subgroup generated by $x_{1}, x_{2}, \cdots\left[x_{1}, x_{2}, \cdots, x_{n}\right]$ is the simple $n$-fold commutator defined inductively by: $[x, y]=x^{-1} y^{-1} x y,\left[x_{1}, \cdots, x_{n}\right]=$ $\left[\left[x_{1}, \cdots, x_{n-1}\right], x_{n}\right](n \geqq 3)$.

Let $X, Y, X_{1}, X_{2}, \cdots$ be subgroups of $G . X \leqq Y(X \unlhd Y)$ means that $X$ is a subgroup (normal subgroup) of $Y ; X<Y(X \triangleleft Y)$ excludes equality. $\mathscr{N}_{G}(X)$ (or $\mathscr{N}(X)$ if no confusion can arise) is the normaliser of $X$ in $G .\left\langle X_{1}, X_{2}, \cdots\right\rangle$ or $X_{1} \cup X_{2} \cup \cdots$ denotes the subgroup generated by $X_{1}, X_{2}, \cdots,\left[X_{1}, X_{2}, \cdots, X_{n}\right]$ is the simple $n$-fold commutator defined inductively by: $[X, Y]=\langle[x, y] \mid x \in X, y \in Y\rangle,\left[X_{1}, \cdots, X_{n}\right]=$ $\left[\left[X_{1}, \cdots, X_{n-1}\right], X_{n}\right](n \geqq 3)$.

$G=G_{(1)} \geqq G_{(2)} \geqq \cdots$ denotes the descending central series of $G$. $G^{\prime}=G_{(2)}=[G, G]$ is the commutator group of $G . \Phi(G)$ is the Frattini 10).

2 A similar result for free polynilpotent groups has been given by Smel'kin [10] (theorem

Spring [11] cites a 6-generator counterexample without carrying through the proof.

- See G. Higman [3], M. Lazard [6]. 
subgroup, $\mathscr{Z}(G)$ the centre, of $G . G^{m}$ denotes the subgroup generated by the $m$-th powers of the elements of $G$.

Homomorphisms $\phi, \psi \cdots$ of groups or lattices will be written exponentially: $x^{\phi \psi}=\left(x^{\phi}\right)^{\downarrow}$. If $\phi: G \rightarrow H$ is a (group) epimorphism and $B / A$ a section of $G$, the equations $(x A)^{\phi^{\prime}}=(x A)^{\phi}(x \in B)$ define an epimorphism $\phi^{\prime}: B \mid A \rightarrow B^{\phi} / A^{\phi}$; similarly, if $\psi$ is an $\mathscr{N} \mathscr{L}$-isomorphism of $F$ onto $H$, the equations $(X / A)^{\psi^{\prime}}=X^{\psi} / A^{\psi}(X / A \in \mathscr{L}(B / A))$ define an $\mathscr{N} \mathscr{L}$-isomorphism $\psi^{\prime}$ of $B / A$ onto $B^{\psi} / A^{\psi}$. It is convenient to call $\phi^{\prime}, \psi^{\prime}$ the restrictions of $\phi, \psi$ to $B / A$. We say that an $\mathscr{L}$-isomorphism $\psi$ of $G$ onto $H$ is induced by an isomorphism if there exists a group isomorphism $\phi: G \rightarrow H$ such that $X^{\phi}=X^{\psi}$ whenever $X \in \mathscr{L}(G)$.

\section{1. $\mathscr{N} \mathscr{L}$-isomorphisms}

An $\mathscr{L}$-isomorphism $\phi: \mathscr{L}(G) \rightarrow \mathscr{L}(H)$ is called

(a) normaliser preserving if $\mathscr{N}(A)^{\phi}=\mathscr{N}\left(A^{\phi}\right)$ for all $A \leqq G$,

(b) commutator preserving if $\left(A^{\prime}\right)^{\phi}=\left(A^{\phi}\right)^{\prime}$ for all $A \leqq G$,

(c) mixed commutator preserving if $[A, B]^{\phi}=\left[A^{\phi}, B^{\phi}\right]$ for all $A, B \leqq G$.

THEOREM 1. The properties (a), (b), (c) of an $\mathscr{L}$-isomorphism are all equivalent.

PROOF. (a) $\Rightarrow$ (b). If $A^{\prime} \leqq B \leqq A \leqq G$, then $\mathscr{N}(B) \geqq A$ and so $\mathscr{N}(B)^{\phi}=\mathscr{N}(B)^{\phi} \geqq A^{\phi}$. Thus every subgroup of $A^{\phi} /\left(A^{\prime}\right)^{\phi}$ is normal. Hence $A^{\phi} /\left(A^{\prime}\right)^{\phi}$ is abelian, or $A^{\phi} /\left(A^{\prime}\right)^{\phi}$ has a subgroup isomorphic to the quaternion group, which is impossible since the latter is not $\mathscr{L}$-isomorphic to an abelian group. Therefore $\left(A^{\prime}\right)^{\phi} \geqq\left(A^{\phi}\right)^{\prime}$. Similarly

$$
\left(\left(A^{\phi}\right)^{\prime}\right)^{\phi^{-1}} \geqq\left(A^{\phi \phi^{-1}}\right)^{\prime}=A^{\prime},
$$

and so, by applying $\phi$ to this we have $\left(A^{\phi}\right)^{\prime} \geqq\left(A^{\prime}\right)^{\phi}$. Hence $\left(A^{\prime}\right)^{\phi}=\left(A^{\phi}\right)^{\prime}$.

(b) $\Rightarrow$ (c). Since

$$
[A, B]=\cup\{[X, Y] \mid X \leqq A, Y \leqq B, X, Y \text { cyclic }\},
$$

it is sufficient to consider the case $A, B$ cyclic. But if $A, B$ are cyclic, the formula

$$
x^{-\alpha}\left[x^{\lambda}, y^{\mu}\right] x^{\alpha}=\left[x^{\lambda+\alpha}, y^{\mu}\right]\left[x^{\alpha}, y^{\mu}\right]^{-1}
$$

shows that $[A, B] \unlhd A \cup B$ and thus that $[A, B]=(A \cup B)^{\prime}$.

(c) $\Rightarrow$ (a). $B \leqq \mathscr{N}(A)$ if and only if $[A, B] \leqq A$.

CoRollary 1. $\mathscr{N} \mathscr{L}$-isomorphisms preserve central and derived series (and thus solubility, derived length, nilpotency, class of nilpotency, etc.). 
The next corollary is a known result, due to Pekelis [7] in the torsionfree case and to Spring [11] in the prime exponent case. Our theorem gives a simple unified proof.

COROLLARY 2. Let $G$ be a locally milpotent group, and $\phi$ an $\mathscr{L}$-isomorphism of $G$ onto a group $H$. If

either (a) $G$ is torsion-free

or (b) $G$ has prime exponent $p$ and is non-abelian

then $\phi$ is an $\mathcal{N} \mathscr{L}$-isomorphism.

Proor. By the proof of the theorem, it is sufficient to show that $\left(M^{\prime}\right)^{\phi}=\left(M^{\phi}\right)^{\prime}$ for each 2-generator subgroup $M$ of $G$. Notice that $M$ is nilpotent. Set $N=M^{\phi}$.

Suppose first that $G$ has exponent $p$. Then $M$ is a finite $p$-group. Hence, if $M$ is non-abelian, $N$ must be a $p$-group (Suzuki [12], thm. 12, p. 12). The same conclusion holds when $M$ is abelian for $M$ is contained in a non-abelian finitely generated subgroup of $G$. It is clear that $N$ is finite and has exponent $p$. Hence $\left(M^{\prime}\right)^{\phi}=(\Phi(M))^{\phi}=\Phi(N)=N^{\prime}$.

Suppose secondly that $G$ is torsion-free. Then $H$ is torsion-free. A torsion-free group is abelian if and only if its subgroup lattice is modular (Suzuki [12], prop. 1.12, p. 19). Thus $\mathscr{Z}(M)^{\phi}=\mathscr{Z}(N)$. Let $1<\mathscr{Z}_{1}(M)<\cdots<\mathscr{Z}_{c}(M)=M$ be the ascending central series of $M$. Since the factor groups $M / \mathscr{Z}_{i}(M)$ are torsion-free the same argument shows that $\mathscr{Z}_{i}(M)^{\phi}=\mathscr{Z}_{i}(N)$ for all $i$. In particular, $N=\mathscr{Z}_{c}(N)$ whence $H$ is nilpotent.

If $M$ is abelian, then $N$ is also abelian because $\mathscr{Z}(M)=\mathscr{Z}(N)$; in this case, $\left(M^{\prime}\right)^{\phi}=N^{\prime}=1$. If $M$ is non-abelian, then $M / \mathscr{X}_{0-2}(M)$ is also non-abelian and so $M / \mathscr{Z}_{c-1}(M)$ is not cyclic. On the other hand, $M / \mathscr{Z}_{c-1}(M)$ is torsion-free and a homomorphic image of the 2-generator group $M / M^{\prime}$. Hence $M^{\prime}=\mathscr{Z}_{c-1}(M)$. Similarly $N^{\prime}=\mathscr{Z}_{\mathrm{c}-1}(N)$. Hence $\left(M^{\prime}\right)^{\phi}=N^{\prime}$, as required.

The following inductive principle is useful for the construction of $\mathscr{N} \mathscr{L}$-isomorphisms.

Lemma 1. Let $G, H$ be finite ${ }^{5}$ groups and $\alpha$ a mapping of $\mathscr{L}(G)$ into $\mathscr{L}(H)$. Then $\alpha$ is an $\mathscr{N} \mathscr{L}$-isomorphism if and only if

(i) $\propto$ maps the interval of $\mathscr{L}(G)$ with end points $\Phi(G)$, G lattice-isomorphically onto the interval of $\mathscr{L}(H)$ with end points $\Phi(H), H$;

(ii) for each maximal subgroup $M$ of $G$, the restriction of a to $\mathscr{L}(M)$ is an $\mathscr{N} \mathscr{L}$-isomorphism of $M$ onto $M^{\alpha}$;

(iii) $\left(G^{\prime}\right)^{\alpha}=H^{\prime}$.

- It is sufficient to assume that each subgroup of $G, H$ is contained in a maximal subgroup. 
Proof. The conditions are clearly necessary. Let us prove that they are suffjcient.

(a) $\alpha$ is surjective. Let $A \in \mathscr{L}(H)$. If $A=H$, then $A=G^{\alpha}$ by (i). If $A<H$, let $B$ be a maximal subgroup of $H$ containing $A$. By (i), $B=C^{\alpha}$ for some maximal subgroup $C$ of $G$. Then, by (ii), $A=D^{\alpha}$ for some $D \in \mathscr{L}(C)$.

(b) $\alpha$ is injective. Suppose $A^{\alpha}=B^{\alpha}$, where $A, B \in \mathscr{L}(G)$. We may assume that $A<G$. Let $C$ be a maximal subgroup of $G$ containing $A$. By (ii) and (i), $A^{\alpha} \leqq C^{\alpha}<G^{\alpha}=H$. Since $B^{\alpha}=A^{\alpha}, B<G$. Let $D$ be a maximal subgroup of $G$ containing $B$. By (ii), $B^{\alpha} \leqq D^{\alpha}$, so that $B^{\alpha} \leqq C^{\alpha} \cap D^{\alpha}$. By (i), $C^{\alpha} \cap D^{\alpha}=(C \cap D)^{\alpha}$ and so $B^{\alpha} \leqq(C \cap D)^{\alpha}$. By (ii) (applied to $\mathscr{L}(D)$ ), $B \leqq C \cap D$. By (ii) (applied to $\mathscr{L}(C)$ ), $B=A$.

(c) $\alpha$ is an $\mathscr{L}$-isomorphism. It is sufficient to show that $A \leqq B \Leftrightarrow A^{\alpha} \leqq B^{\alpha}$ for $A, B \in \mathscr{L}(G)$. Since $\alpha^{-1}$ exists (by (a), (b)) and clearly satisfies the conditions of the theorem, it is sufficient to show that $A \leqq B \Rightarrow A^{\alpha} \leqq B^{\alpha}$. If $B=G$, then $A^{\alpha} \leqq H=B^{\alpha}$. If $B<G$, let $C$ be a maximal subgroup of $G$ containing $B$. Then $A^{\alpha} \leqq B^{\alpha}$ by (ii) applied to $\mathscr{L}(C)$.

(d) $\propto$ is an $\mathscr{N} \mathscr{L}$-isomorphism. By theorem 1 , it suffices to prove that $\left(A^{\prime}\right)^{\alpha}=\left(A^{\alpha}\right)^{\prime}$ for $A \in \mathscr{L}(G)$. If $A=G$, this follows from (iii). If $A<G$, let $B$ be a maximal subgroup of $G$ containing $A$. Then $\left(A^{\prime}\right)^{\alpha}=\left(A^{\alpha}\right)^{\prime}$ by theorem 1 and (ii) applied to $\mathscr{L}(B)$.

The following is the analogue of theorem 1 for Lie algebras.

THEOREM 1'. Let $L, M$ be Lie algebras over the same principal domain $R$. Let $\phi: \mathscr{L}(L) \rightarrow \mathscr{L}(M)$ be an isomorphism of the lattice $\mathscr{L}(L)$ of all subalgebras of $L$ onto $\mathscr{L}(M)$. Then the following are equivalent:

(a) $\mathscr{N}(A)^{\phi}=\mathscr{N}\left(A^{\phi}\right)$ for all subalgebras $A \leqq L$,

(b) $\left(A^{\prime}\right)^{\phi}=\left(A^{\phi}\right)^{\prime}$ for all $A \leqq L$.

Proof. (a) $\Rightarrow$ (b). This follows as in theorem 1 once we have proved: if every subalgebra of $L$ is an ideal, then $L$ is abelian.

To prove this, let $a, b \in L$. Then $a, b$ belong to an $R$-submodule $R x_{1} \oplus R x_{2} \oplus \cdots \oplus R x_{n}$. Each $R x_{i}$ is a subalgebra of $L$ and is therefore an ideal. For $i \neq j, x_{i} x_{j} \in R x_{i} \cap R x_{j}=0$. Thus

$$
\left(\alpha_{1} x_{1}+\cdots+\alpha_{n} x_{n}\right)\left(\beta_{1} x_{1}+\cdots+\beta_{n} x_{n}\right)=0 \text { for all } \alpha_{i}, \beta_{i} \in R
$$

and in particular, $a b=0$.

(b) $\Rightarrow$ (a). This is obvious because, for $A \leqq L, x \in L$,

$$
R x \leqq \mathcal{N}(A) \Leftrightarrow(R x \cup A)^{\prime} \leqq A .
$$

CoRollaRy $1^{\prime}$. $\mathscr{N} \mathscr{L}$-isomorphisms of Lie algebras preserve central and derived series. 
COROllary 2'. Let $L, M$ be nilpotent Lie algebras over a field. Then every $\mathscr{L}$-isomorphism $\phi: \mathscr{L}(L) \rightarrow \mathscr{L}(M)$ is $\mathscr{N} \mathscr{L}$.

There is no equivalent for Lie algebras of part (c) of theorem 1, for $(R x \cup R y)^{\prime}$ does not in general coincide with $R x y(x, y \in L)$. In corollary $2^{\prime}$, it is essential to assume both algebras nilpotent, as it is possible for a nilpotent algebra to be $\mathscr{L}$-isomorphic to a non-nilpotent algebra (for example, the two 2 -dimensional Lie algebras are $\mathscr{L}$-isomorphic).

\section{2. $\mathscr{N} \mathscr{L}$-isomorphisms between non-isomorphic p-groups}

Let $p$ be a prime $>2$. We give examples of $\mathcal{N} \mathscr{L}$-isomorphisms between non-isomorphic finite $p$-groups. By means of Lazard's theory [6], these examples can be turned into examples of the same phenomenon for Lie rings; and when the groups have exponent $p$ the Lie rings become Lie algebras over the field of $p$ elements.

Wiman [13] and Blackburn ${ }^{6}$ [2] have determined those $p$-groups of maximal class ${ }^{7}$ which contain an abelian subgroup of index $p$. Such a group $G$, of order $p^{n}(n \geqq 3)$, is isomorphic to an abstract group $A_{n}(\gamma, \delta)$ defined by generators $s, s_{1}, s_{2}, \cdots$ and relations

$$
\begin{cases}{\left[s_{i}, s\right]=s_{i+1}} & \left(i \geqq 1 ; s_{n}=s_{n+1}=\cdots=1\right), \\ {\left[s_{i}, s_{i}\right]=1,} & (i, j \geqq 1), \\ s^{p}=s_{n-1}^{\delta}, & \\ s_{1}^{p} s_{2}^{\left(\frac{1}{2}\right)} \cdots s_{p}=s_{n-1}^{\gamma}, & \\ s_{i}^{p} s_{i+1}^{(p)} \cdots s_{i+p-1}=1 & (i \geqq 2) .\end{cases}
$$

Set $B_{n}(\gamma)=A_{n}(\gamma, 0)$. Then $B_{n}(\gamma) \cong B_{n}\left(\gamma^{\prime}\right)$ if and only if the congruence $\gamma x^{n-2} \equiv \gamma^{\prime}(\bmod p)$ has a solution $x \neq \equiv 0(\bmod p)($ Blackburn, 1.c.). On the other hand, we now prove that if (a) $\gamma \gamma^{\prime} \neq 0(\bmod p)$ and (b) when $p=n-1$, $(\gamma-1)\left(\gamma^{\prime}-1\right) \not \equiv 0(\bmod p)$, then there exists an $\mathscr{N} \mathscr{L}$-isomorphism of $B_{n}(\gamma)$ onto $B_{n}\left(\gamma^{\prime}\right)$.

Since the result is trivial for $n=3$, we assume $n \geqq 4$. Let $s, s_{1}, \cdots$ and $s^{\prime}, s_{1}^{\prime}, \cdots$ be the generators of $G=B_{n}(\gamma)$ and $H=B_{n}\left(\gamma^{\prime}\right)$ respectively. Choose an integer $k$ such that $\gamma \equiv k \gamma^{\prime}(\bmod p)$. Then the maximal subgroups of $G, H$ are

and

$$
\begin{aligned}
& G_{\lambda}=\left\langle s s_{1}^{\lambda}, s_{2}, \cdots, s_{n-1}\right\rangle \\
& G_{p}=\left\langle s_{1}, s_{2}, \cdots, s_{n-1}\right\rangle,
\end{aligned}
$$

- The reader is referred to section 4 of Blackburn's paper for the properties of maximal class groups used here.

T The idea of looking at maximal class groups for examples was suggested to us by J. L. Alperin. 


$$
\begin{array}{ll}
H_{\lambda}=\left\langle s^{\prime} s_{j}^{\prime k \lambda}, s_{2}^{\prime}, \cdots, s_{n-1}^{\prime}\right\rangle & (0 \leqq \lambda<p), \\
H_{p}=\left\langle s_{1}^{\prime}, s_{2}^{\prime}, \cdots, s_{n-1}^{\prime}\right\rangle . &
\end{array}
$$

For $\lambda<p$, the generators $\sigma=s s_{1}^{\lambda}, \sigma_{1}=s_{2}, \cdots, \sigma_{n-2}=s_{n-1}$ of $G_{\lambda}$, and the generators $\sigma^{\prime}=s^{\prime} s_{1}^{\prime k \lambda}, \sigma_{1}^{\prime}=s_{2}^{\prime}, \cdots, \sigma_{n-2}^{\prime}=s_{n-1}^{\prime}$ of $H_{\lambda}$, satisfy the defining relations for $A_{n-1}(0, \lambda \gamma)$. Hence there is an isomorphism

$$
\alpha_{\lambda}: G_{\lambda} \rightarrow H_{\lambda}
$$

such that $\left(s s_{1}^{\lambda}\right)^{\alpha_{\lambda}}=s^{\prime} s_{1}^{\prime k \lambda}, s_{i}^{\alpha_{\lambda}}=s_{i}^{\prime}(2 \leqq i \leqq n-1)$.

$G_{p}$ is an abelian group whose defining relations are the last two lines of (2.1). $H_{n}$ is an abelian group with the same defining relations, except that $\gamma^{\prime}$ replaces $\gamma$. We show that $H_{p}=\left\langle s_{1}^{*}, s_{2}^{\prime} \cdots, s_{n-1}^{\prime}\right\rangle$, where $\left(s_{1}^{*}\right)^{p} s_{2}^{\prime(z)} \cdots s_{p}^{\prime}=s_{n-1}^{\prime \gamma}$. It will follow that there is an isomorphism

$$
\alpha_{p}: G_{p} \rightarrow H_{p}
$$

such that $s_{1}^{\alpha}=s_{1}^{*}, s_{i}^{\alpha}=s_{i}^{\prime}(2 \leqq i \leqq n-1)$.

For $p>n-1$, the defining relations of $H_{p}$ reduce to $s_{1}^{\prime p}=s_{n-1}^{\prime \gamma^{\prime}}$, $s_{i}^{\prime p}=1(i \geqq 2)$. In this case we may take $s_{1}^{*}=s_{1}^{\prime k}$, since $s_{1}^{* p}=s_{1}^{\prime k p}=s_{n-1}^{\prime k \gamma^{\prime}}=$ $s_{n-1}^{\prime \gamma}$. For $p=n-1$, the defining relations are the same, except that $s_{1}^{\prime p}=s_{n-1}^{\prime\left(\gamma^{\prime}-1\right)}$. A similar choice of $s_{1}^{*}$ is possible by our assumption (b). Finally, if $p<n-1$, then $s_{n-p}^{\prime p} s_{n-p+1}^{(p)} \cdots s_{n-2}^{\prime p}=s_{n-1}^{\prime-1}$ so that $s_{n-1}^{\prime}=\sigma^{p}$, where $\sigma \in\left\langle s_{2}^{\prime}, \cdots, s_{n-1}^{\prime}\right\rangle$. We may therefore take $s_{1}^{*}=s_{1}^{\prime} \sigma^{\gamma-\gamma^{\prime}}$.

We now define a mapping $\alpha: \mathscr{L}(G) \rightarrow \mathscr{L}(H)$ by:

$$
\begin{aligned}
G^{\alpha} & =H, \\
K^{\alpha} & =K^{\alpha_{\mu}} \text { if } K \leqq G_{\mu} \quad(0 \leqq \mu \leqq p) .
\end{aligned}
$$

Since the isomorphisms $\alpha_{\mu}$ all agree on $\left\langle s_{2}, \cdots, s_{n-1}\right\rangle$, the definition is unambiguous. All the conditions of lemma 1 are satisfied, so that $\alpha$ is an $\mathscr{N} \mathscr{L}$-isomorphism.

The simplest examples of non-isomorphic, $\mathscr{N} \mathscr{L}$-isomorphic pairs provided by our results are:

for $p>3$, the groups $B_{4}(1), B_{4}(k)$ of order $p^{4}$, where $k$ is a nonquadratic residue $(\bmod p)$;

for $p=3$, the groups $B_{5}(1), B_{5}(-1)$ of order $3^{5}$.

The groups in the above examples have exponent $p^{k}>p$. We now give examples of $\mathscr{N} \mathscr{L}$-isomorphisms between non-isomorphic groups of exponent $p$. The groups in question are again groups of maximal class.

Let $n$ be an integer, and $p$ a prime, such that $8 \leqq n \leqq p$. Let $C_{n}(\varepsilon)$ denote the group of order $p^{n}$ with generators $s, s_{1}, s_{2}, \cdots$ and defining relations 


$$
\begin{array}{ll}
{\left[s_{i}, s\right]=s_{i+1}} & \left(i \geqq 1 ; s_{n}=s_{n+1}=\cdots=1\right), \\
{\left[s_{i}, s_{1}\right]=s_{i+2}^{\varepsilon}} & (i \geqq 2), \\
{\left[s_{i}, s_{j}\right]=1} & (i, j \geqq 2), \\
s^{p}=s_{1}^{p}=1 . &
\end{array}
$$

Since $C_{n}(\varepsilon)$ is generated by $s, s_{1}$ of order $p$ and has class $n-1<p$, it is a group of exponent $p$. We prove that $G=C_{n}(1), H=C_{n}(-1)$ are $\mathscr{N} \mathscr{L}$ isomorphic but not isomorphic.

We take the generators of $G$ to be $s, s_{1}, s_{2}, \cdots$ and those of $H$ to be $s, s_{1}^{\prime}, s_{2}, \cdots$, so that the subgroup $\left\langle s, s_{2}, \cdots,\right\rangle$ of order $p^{n-1}$ is common to both. Set $V=\Phi(G)=\Phi(H)=\left\langle s_{2}, \cdots\right\rangle$. Then, denoting the endomorphisms $v \rightarrow v^{s-1}, v \rightarrow v^{s_{1}-1}, v \rightarrow v^{s_{1}^{\prime}-1}$ of $V$ by $\sigma, \sigma_{1}, \sigma_{1}^{\prime}$, we have

$$
\sigma^{n-2}=0, \sigma^{n-3} \neq 0, \sigma_{1}=-\sigma_{1}^{\prime}=\sigma^{2}
$$

Using these facts, it is easy to see that an $\mathscr{N} \mathscr{L}$-isomorphism $\alpha$ of $G$ onto $H$ is defined as follows:

$G^{\alpha}=H$;

if $K \leqq V, K^{\alpha}=K$;

if $L=K \cap V<K<G$, then $K$ has the form

$$
\left\langle s s_{1}^{\lambda} t, L\right\rangle \text { or }\left\langle s_{1} t, L\right\rangle
$$

and we take

$$
K^{\alpha}=\left\langle s s_{1}^{\prime \lambda} t, L\right\rangle \quad \text { or } \quad\left\langle s_{1}^{\prime} t, L\right\rangle .
$$

Suppose now that there exists an isomorphism $\theta: G \rightarrow H$. If $s^{\theta} V=s^{a} s_{1}^{\theta} V, s_{1}^{\theta} V=s^{\gamma} s_{1}^{\delta} V$, and if $\omega$ is the restriction of $\theta$ to $V$, then

$$
v^{\omega s^{\theta}}=v^{s \omega}, v^{\omega \varepsilon_{1}^{\theta}}=v^{a_{1} \omega} \text { for } v \in V \text {, }
$$

so that

$$
\begin{aligned}
& \omega^{-1} \sigma \omega=(1+\sigma)^{\alpha}\left(1-\sigma^{2}\right)^{\beta}-1 \\
& \omega^{-1} \sigma^{2} \omega=(1+\sigma)^{\gamma}\left(1-\sigma^{2}\right)^{\delta}-1 .
\end{aligned}
$$

Hence

$$
(1+\sigma)^{\gamma}\left(1-\sigma^{2}\right)^{\gamma}-1=\left\{(1+\sigma)^{\alpha}\left(1-\sigma^{2}\right)^{\beta}-1\right\}^{2} .
$$

Comparing coefficients of $\sigma, \sigma^{2}, \sigma^{3}, \sigma^{4}, \sigma^{5}$ on both sides (this is permissible as $\sigma^{n-3} \not \equiv 0$ and $\left.n \geqq 8\right)$, and using the fact that $\alpha \delta-\beta \gamma \neq 0(\bmod p)$, we get in succession the following congruences $(\bmod p)$ : 


$$
\begin{aligned}
& \gamma \equiv 0, \quad-\delta \equiv \alpha^{2}, \quad 0 \equiv\left(\begin{array}{l}
\alpha \\
2
\end{array}\right)-\beta, \\
& \left(\begin{array}{l}
\delta \\
2
\end{array}\right) \equiv 2 \alpha\left(\left(\begin{array}{l}
\alpha \\
3
\end{array}\right)-\alpha \beta\right), \\
& 0 \equiv\left(\begin{array}{l}
\alpha \\
4
\end{array}\right)-\beta\left(\begin{array}{l}
\alpha \\
2
\end{array}\right)+\left(\begin{array}{l}
\beta \\
2
\end{array}\right) .
\end{aligned}
$$

On simplification, the second and third lines yield $7 \alpha^{2} \equiv 1$ and $\alpha^{2} \equiv 1$, giving the contradiction $7 \equiv 1$. Thus ${ }^{8} \mathrm{G} \cong H$.

\section{A lemma on multilinear mappings}

The following lemma plays an essential part in the proof of theorem 2 in the next section.

Lemma 2. Let $A_{1}, \cdots, A_{t}, L, M$ be (additive) abelian groups. Let $f\left(x_{1}, \cdots, x_{t}\right), g\left(x_{1}, \cdots, x_{t}\right)$ be multilinear functions of the variables $x_{i} \in A_{i}$ $(i=1, \cdots, t)$ with values in $L, M$ respectively. Suppose that the values of $f, g$ generate $L, M$ and that there exists an $\mathscr{L}$-isomorphism $\phi$ of $L$ onto $M$ such that

$$
\left.\left\langle f\left(x_{1}, \cdots, x_{t}\right)\right\rangle^{\phi}=\left\langle g\left(x_{1}, \cdots, x_{t}\right)\right\rangle \text { (all } x_{1}, \cdots, x_{t}\right) .
$$

Then there exists an isomorphism $\alpha$ of $L$ onto $M$ such that

$$
f\left(x_{1}, \cdots, x_{t}\right)^{\alpha}=g\left(x_{1}, \cdots, x_{t}\right) \quad\left(\text { all } x_{1}, \cdots, x_{t}\right) .
$$

Remark 1. The expected generalisation to $R$-modules, $R$ a principal ideal domain, is valid and can be proved by the same method. E.g., when the $A_{i}$ are vector spaces over a field $R$ and $L=M=R$, the generalisation states that multilinear forms which vanish for the same values of the variables are scalar multiples of one another.

Remark 2. The conclusion of the lemma is equivalent to the following statement:

$$
f(x)+f(y)+\cdots=0 \text { if and only if } g(x)+g(y)+\cdots=0 .
$$

(Here $x$ denotes a row of variables $x_{1}, \cdots, x_{t}$ and so on.) In fact, if the condition holds,

$$
f(x)+f(y)+\cdots \leftrightarrow g(x)+g(y)+\cdots
$$

is a well defined $1-1$ correspondence; it is then clearly an isomorphism.

The lemma is proved by induction on $t$. If $t=1, f, g$ are homomorphisms of $A_{1}$ onto $L, M$ with the same kernel, so that $f(x) \leftrightarrow g(x)$ is an isomorphism

- The same conclusion holds for $n=7$ provided that the congruence $7 \alpha^{2} \equiv 1(\bmod p)$ has no solution, i.e. if $(7 / p) \neq 1$. 
between $L, M$. We assume now that $t>1$ and that the lemma holds for a smaller number of variables.

By remark 2, and since a given sum $f(x)+f(y)+\cdots$ involves only finitely many values of the variables $x_{i}, y_{j}, \cdots$, it is sufficient to prove the lemma when the $A_{i}$ are finitely generated. Then $L, M$ are also finitely generated. Hence there exists a family of subgroups $\left(L_{i}\right)$ of $L$ such that (a) each factor group $L / L_{i}$ is cyclic of prime power order and (b) $\bigcap_{i} L_{i}=0$. Define

$$
\begin{aligned}
& f_{i}(x)=f(x)+L_{i} \quad\left(\in L / L_{i}\right) \\
& g_{i}(x)=g(x)+M_{i} \quad\left(\in M / M_{i}\right),
\end{aligned}
$$

where $M_{i}=L_{i}^{\phi}$. Then $f_{i}, g_{i}$ are multilinear mappings whose values generate $L / L_{i}, M / M_{i}$ and $\left\langle f_{i}(x)\right\rangle^{\phi_{i}}=\left\langle g_{i}(x)\right\rangle$, where $\phi_{i}$ is the restriction of $\phi$ to $L / L_{i}$. Thus, if the lemma holds for each pair $f_{i}, g_{i}$, we have

$$
f(x)+f(y)+\cdots \in L_{i} \Leftrightarrow g(x)+g(y)+\cdots \in M_{1}
$$

for each $i$. Therefore, since $\bigcap_{i} L_{i}=0$ and $\bigcap_{i} M_{i}=0$,

$$
f(x)+f(y)+\cdots=0 \Leftrightarrow g(x)+g(y)+\cdots=0,
$$

so that the lemma holds for $f, g$. Hence it is sufficient to prove the lemma when $L$ is cyclic of prime power order.

Suppose $L$ cyclic of order $p^{m}$. Since $\phi$ is an $\mathscr{L}$-isomorphism, $M$ is cyclic of order $q^{m}$ for some prime $q$. Now $\langle k f(x)\rangle^{\phi}=\left\langle f\left(k x_{1}, x_{2}, \cdots,\right)\right\rangle^{\phi}=$ $\left\langle g\left(k x_{1}, x_{2}, \cdots\right)\right\rangle=\langle k g(x)\rangle$, so that $f(x), g(x)$ have the same order. Hence $q=p$. We may therefore assume that $L=M$.

The assertion of the lemma in this case is that

$$
f(x)=k g(x) \text { for all } x,
$$

where $k$ is a fixed integer prime to $p$. Let $r_{x}$ denote the order of $f(x)$. We know, since $g(x)$ also has order $r_{x}$, that

$$
f(x)=k_{x} g(x),
$$

where $k_{x}$ is prime to $p$. It is sufficient to prove that

$$
k_{x} \equiv k_{y}\left(\bmod r_{v}\right) \quad \text { whenever } \quad r_{x} \geqq r_{y}>1 .
$$

For then (3.I) will hold with $k=k_{\bar{x}}$, where $\bar{x}$ is such that $r_{\bar{x}}$ has the largest possible value.

Consider the homomorphisms $\alpha, \beta: A_{t} \rightarrow L$ defined by

$$
a^{\alpha}=f\left(x_{1}, \cdots, x_{t-1}, a\right), \quad a^{\beta}=f\left(y_{1}, \cdots, y_{t-1}, a\right) .
$$

Since $\alpha, \beta$ are non-zero, the inverse images $\alpha^{-1}\left(p A_{t}^{\alpha}\right)$ and $\beta^{-1}\left(p A_{t}^{\beta}\right)$ are subgroups of $A_{1}$ of index $p$. An abelian group cannot be the set-theoretical 
union of two proper subgroups. Therefore we can choose $a \in A_{t}$ such that $a \notin \alpha^{-1}\left(p A_{t}^{\alpha}\right)$ and $a \notin \beta^{-1}\left(p A_{t}^{\beta}\right)$. Then $a^{\alpha} \notin p A_{t}^{\alpha}$ and $a^{\beta} \notin p A_{t}^{\beta}$, so that

$$
\left\langle a^{\alpha}\right\rangle=A_{t}^{\alpha}, \quad\left\langle a^{\beta}\right\rangle=A_{t}^{\beta} .
$$

Thus, if $u$ and $v$ denote the rows of variables $x_{1}, \cdots, x_{t-1}, a$ and $y_{1}, \cdots, y_{t-1}, a$, we have

and therefore

$$
A_{t}^{\alpha}=\langle f(u)\rangle, \quad A_{t}^{\beta}=\langle f(v)\rangle
$$

$$
r_{u} \geqq r_{x}, \quad r_{v} \geqq r_{y} .
$$

Let us now apply the lemma in the case $t=1$ to the linear mappings $\alpha, \alpha^{\prime}: A_{t} \rightarrow L$ given by

$$
\alpha(a)=f\left(x_{1}, \cdots, x_{t-1}, a\right), \quad \alpha^{\prime}(a)=g\left(x_{1}, \cdots, x_{t-1}, a\right) .
$$

We deduce that

$$
k_{u} \equiv k_{x}\left(\bmod r_{x}\right)
$$

since $r_{u} \geqq r_{x}$. Similarly

$$
k_{\mathrm{v}} \equiv k_{\mathrm{v}}\left(\bmod r_{y}\right) \text {. }
$$

Finally, let us apply the lemma to the multilinear forms

$$
\begin{aligned}
& \chi\left(z_{1}, \cdots, z_{t-1}\right)=f\left(z_{1}, \cdots, z_{t-1}, a\right), \\
& \psi\left(z_{1}, \cdots, z_{t-1}\right)=g\left(z_{1}, \ldots, z_{t-1}, a\right) .
\end{aligned}
$$

We deduce that

$$
k_{u} \equiv k_{v}(\bmod \rho),
$$

where $\rho$ is the smaller of $r_{u}, r_{v}$. Putting together the results (3.3)-(3.6), we get (3.2) as required.

\section{The main results}

We study properties of a fixed $\mathscr{N} \mathscr{L}$-isomorphism between two groups $G, H$. It is convenient for this purpose to represent $G, H$ as factor groups of an auxiliary group $F$. In the applications, $F$ will be a suitable free, or relatively free, group.

Let $\phi: \mathscr{L}(G) \rightarrow \mathscr{L}(H)$ be an $\mathscr{N} \mathscr{L}$-isomorphism and $\lambda: F \rightarrow G$, $\mu: F \rightarrow H$ epimorphisms. We say that $\phi$ is compatible with the pair $\lambda, \mu$ if $K^{\lambda \phi}=K^{\mu}$ for all $K \in \mathscr{L}(F)$. When this is the case, the equations $\left(x^{\lambda}\right)^{\theta}=x^{\mu}$ $(x \in F)$ clearly define an isomorphism $\theta: G \rightarrow H$ and $\phi$ is the $\mathscr{L}$-isomorphism induced by $\theta$.

More generally, we say that $\phi$ is compatible with the pair $\lambda, \mu$ on the 
section $P / Q$ of $F$ if $K^{\lambda \phi}=K^{\mu}$ whenever $Q \leqq K \leqq P$, i.e. if the restriction of $\phi$ to $P^{\lambda} / Q^{\lambda}$ maps $P^{\lambda} / Q^{\lambda}$ onto $P^{\mu} / Q^{\mu}$ and is compatible with the restrictions $\lambda^{\prime}, \mu^{\prime}$ of $\lambda, \mu$ to $P / Q$.

THEOREM 2. Let $\lambda: F \rightarrow G, \mu: F \rightarrow H$ be epimorphisms and $\phi: \mathscr{L}(G) \rightarrow$ $\mathscr{L}(H)$ an $\mathscr{N} \mathscr{L}$-isomorphism. Let $P_{1} / Q_{1}, \cdots, P_{t} / Q_{t}$ be central sections of $F$ on each of which $\phi$ is compatible with the pair $\lambda, \mu$. Let

$$
\begin{aligned}
& P=\left[P_{1}, \cdots, P_{t}\right] \\
& Q=\left[Q_{1}, P_{2}, \cdots, P_{t}\right]\left[P_{1}, Q_{2}, \cdots, P_{t}\right] \cdots\left[P_{1}, P_{2}, \cdots, Q_{t}\right] .
\end{aligned}
$$

Then $P / Q$ is a central section of $F$ and $\phi$ is compatible with the pair $\lambda, \mu$ on $P / Q$.

Proof. We prove the theorem for two sections $P_{1} / Q_{1}, P_{2} / Q_{2}$. The theorem for $t$ sections then follows in an obvious way by induction.

Since

$$
\begin{aligned}
{[P, G]=\left[P_{1}, P_{2}, G\right] } & \leqq\left[G, P_{1}, P_{2}\right]\left[P_{2}, G, P_{1}\right] \\
& \leqq\left[Q_{1}, P_{2}\right]\left[Q_{2}, P_{1}\right]=Q,
\end{aligned}
$$

$P / Q$ is a central section. Let $u, v \in P_{1}, x, y \in P_{2}$. Since $P / Q \leqq \mathscr{Z}(G / Q)$, we have

$$
[u v, x]=v^{-1}[u, x] v[v, x] \equiv[u, x][v, x](\bmod Q)
$$

Similarly,

$$
[u, x y] \equiv[u, x][u, y](\bmod Q) .
$$

Taking $v \in Q_{1}$ and $y \in Q_{2}$, we deduce that the coset of $Q$ containing $[u, x]$ depends only on the cosets of $Q_{1}, Q_{2}$ containing $u, x$. Hence

$$
\begin{aligned}
& f\left(u Q_{1}, x Q_{2}\right)=\left[u^{\lambda}, x^{\lambda}\right] Q^{\lambda}, \\
& g\left(u Q_{1}, x Q_{2}\right)=\left[u^{\mu}, x^{\mu}\right] Q^{\mu},
\end{aligned}
$$

are bilinear functions of the variables $u Q_{1} \in P_{1} / Q_{1}$ and $x Q_{2} \in P_{2} / Q_{2}$ with values in $P^{\lambda} / Q^{\lambda}, P^{\mu} / Q^{\mu}$ respectively. It is clear that the values of $f, g$ generate $P^{\lambda} / Q^{\lambda}, P^{\mu} / Q^{\mu}$.

Now, since $\phi$ is mixed commutator preserving and is compatible with the pair $\lambda, \mu$ on each of $P_{1} / Q_{1}, P_{2} / Q_{2}$, we have

and

$$
P^{\lambda \phi}=P^{\mu}, Q^{\lambda \phi}=Q^{\mu},
$$

$$
\begin{aligned}
\langle[u, x], Q\rangle^{\lambda \phi} & =\left\langle\left[\left\langle u, Q_{1}\right\rangle,\left\langle x, Q_{2}\right\rangle\right], Q\right\rangle^{\lambda \phi} \\
& =\left\langle\left[\left\langle u, Q_{1}\right\rangle^{\lambda \phi},\left\langle x, Q_{2}\right\rangle^{\lambda \phi}\right], Q^{\lambda \phi}\right\rangle \\
& =\left\langle\left[\left\langle u, Q_{1}\right\rangle^{\mu},\left\langle x, Q_{\varepsilon}\right\rangle^{\mu}\right], Q^{\mu}\right\rangle \\
& =\langle[u, x], Q\rangle^{\mu} .
\end{aligned}
$$


Thus the restriction, $\phi^{\prime}$, of $\phi$ to $P^{\lambda} / Q^{\lambda}$ maps $P^{\lambda} / Q^{\lambda}$ onto $P^{\mu} / Q^{\mu}$ and satisfies $\left\langle f\left(u Q_{1}, x Q_{2}\right)\right\rangle^{\phi^{\prime}}=\left\langle g\left(u Q_{1}, x Q_{2}\right)\right\rangle$. By lemma 1 , there is an isomorphism $\alpha: P^{\lambda} / Q^{\lambda} \rightarrow P^{\mu} / Q^{\mu}$ such that $f\left(u Q_{1}, x Q_{2}\right)^{\alpha}=g\left(u Q_{1}, x Q_{2}\right)$, i.e. $([u, x] Q)^{\lambda \alpha}=$ $([u, x] Q)^{\mu}$. Hence, if $Q \leqq K \leqq P$, we have $\left(K^{\lambda} / Q^{\lambda}\right)^{\alpha}=\left(K^{\mu} / Q^{\mu}\right)$ and so, since $\alpha$ induces $\phi^{\prime},\left(K^{\lambda} / Q^{\lambda}\right)^{\phi^{\prime}}=K^{\mu} / Q^{\mu}$. Thus $K^{\lambda \phi}=K^{\mu}$, which proves the theorem.

Notation. If $\phi: \mathscr{L}(G) \rightarrow \mathscr{L}(H)$ is an $\mathscr{N} \mathscr{L}$-isomorphism, let $\phi_{i}: \mathscr{L}\left(G_{(i)} / G_{(i+1)}\right) \rightarrow \mathscr{L}\left(H_{(i)} / H_{(i+1)}\right)$ denote its restriction to the $i$-th factor $G_{(2)} / Q_{(i+1)}$ of the descending central series $(i=1,2, \cdots)$.

Corollary. Let $\phi: \mathscr{L}(G) \rightarrow \mathscr{L}(H)$ be an $\mathscr{N} \mathscr{L}$-isomorphism such that $\phi_{1}: \mathscr{L}\left(G / G^{\prime}\right) \rightarrow \mathscr{L}\left(H / H^{\prime}\right)$ is induced by an isomorphism $\theta: G / G^{\prime} \rightarrow H / H^{\prime}$. Then every $\phi_{i}$ is induced by an isomorphism.

Proof. We may of course assume $G$ and $H$ nilpotent. Represent $G$ as a factor group $F / M$ of a free group $F$. Let $\lambda: F \rightarrow F / M=G$, $\alpha: G \rightarrow G / G^{\prime}$ be the canonical epimorphisms. Since $F$ is free, the epimorphism $\lambda \alpha \theta_{1}: F \rightarrow H / H^{\prime}$ can be lifted to a homomorphism $\mu: F \rightarrow H$ such that $H^{\prime} F^{\mu}=H$. Since $H$ is nilpotent, $F^{\mu}=H$. Clearly, $\phi$ is compatible with the pair $\lambda, \mu$ on $F / F^{\prime}$. By the theorem (with all sections $P_{j} / Q_{j}$ equal to $\left.F / F^{\prime}\right), \phi$ is compatible with the pair $\lambda, \mu$ on $F_{(i)} / F_{(i+1)}$. Hence $\phi_{i}$ is induced by an isomorphism.

For the proof of the next theorem we require the following technical result.

LemMa 3. Let $F$ be a finite, relatively free, 2-generator $p$-group of class 3 , and $\phi: \mathscr{L}(F) \rightarrow \mathscr{L}(F)$ an $\mathscr{N} \mathscr{L}$-automorphism of $F$. Let $p^{t}$ be the exponent of $F_{(3)}$. Then the restriction, $\psi$, of $\phi$ to $F / F^{\prime} F^{p^{t}}$ is induced by an automorphism of $F / F^{\prime} F^{p^{*}}$.

Proof. It is not difficult to see that $F$ has generators $x, y$ and defining relations of the form

$$
\begin{aligned}
& x^{p^{p}}=y^{p^{p}}=z^{p^{p}}=u^{p^{p}}=v^{p^{p}}=1 \\
& {[x, u]=[y, u]=[x, v]=[y, v]=1,}
\end{aligned} \quad(r \geqq s \geqq t),
$$

where

$$
z=[x, y], \quad u=[x, z], \quad v=[y, z] .
$$

Replacing $F$ by $F / F^{p^{t}}$, we may assume that $r=s=t$ and $F^{\prime}=F^{\prime} F^{p^{t}}$.

Let $\langle\boldsymbol{x}\rangle^{\phi}=\langle X\rangle,\langle y\rangle^{\phi}=\langle Y\rangle$ and write

$$
Z=[X, Y], \quad U=[X, Z], \quad V=[Y, Z] .
$$

Since $\phi$ is mixed commutator preserving,

$$
\langle z\rangle^{\phi} \equiv\langle Z\rangle\left(\bmod F_{(3)}\right),\langle u\rangle^{\phi}=\langle U\rangle,\langle v\rangle^{\phi}=\langle V\rangle .
$$


Now, since $F^{\prime}$ is the direct product of 3 cyclic groups of order $p^{t}$, the restriction of $\phi$ to $F^{\prime}$ is induced by an automorphism of $F^{\prime}$ (Baer [1]; cf. Suzuki [12], p. 35). Hence

$$
\left\langle u^{\alpha} v^{\beta}\right\rangle^{\phi}=\left\langle U^{m \alpha} V^{n \beta}\right\rangle
$$

for certain fixed integers $m, n$. On the other hand, if

$$
\left\langle x^{\alpha} y^{\beta}\right\rangle^{\phi} \equiv\left\langle X^{\alpha^{\prime}} Y^{\beta^{\prime}}\right\rangle\left(\bmod F^{\prime}\right),
$$

then

$$
\begin{aligned}
\left\langle u^{\alpha} v^{\beta}\right\rangle^{\phi} & =\left\langle\left[x^{\alpha} y^{\beta}, z\right]\right\rangle^{\phi} \\
& =\left\langle\left[X^{\alpha^{\prime}} Y^{\beta^{\prime}}, Z\right]\right\rangle \\
& =\left\langle U^{\alpha^{\prime}} V^{\beta^{\prime}}\right\rangle .
\end{aligned}
$$

Hence $\left\langle x^{\alpha} y^{\beta}\right\rangle^{\phi} \equiv\left\langle X^{m a} Y^{n \beta}\right\rangle\left(\bmod F^{\prime}\right)$, and so $\psi$ is induced by the automorphism $x^{\alpha} y^{\beta} F^{\prime} \rightarrow X^{m \alpha} Y^{n \beta} F^{\prime}$ of $F / F^{\prime}$. This proves the lemma.

Let $V$ be a nilpotent variety (i.e. a variety consisting of nilpotent groups) and $F$ a free group of $V$. Let $c$ be the class of $F$. Then a factor group

$$
G=F / M, \quad M<F_{(\mathrm{c})},
$$

will be called an almost free group of $V$. Notice that the class of $G$ is $c$ and that $G / G_{(\mathrm{c})} \cong F / F_{(\mathrm{c})}$ is relatively free.

THEOREM 3. Let $V$ be a nilpotent variety, $G$ a non-abelian almost free group of $V$. Let $\phi$ be an $\mathscr{N} \mathscr{L}$-isomorphism of $G$ onto a member $H$ of $V$. Then $G \cong H$.

Proof. Represent $G$ in the form (3.1) and let $\lambda: F \rightarrow F / M=G$, $\alpha: F \rightarrow G / G^{\prime}$ be the canonical epimorphisms. Since $G$ is non-abelian, $G / G^{\prime}$ is relatively free and so is a (restricted) direct product of isomorphic cyclic groups. Since $G$ is non-cyclic, the number, $k$, of cyclic factors is at least 2. Therefore $G / G^{\prime} \cong H / H^{\prime}$. Let $\theta$ be any isomorphism of $G / G^{\prime}$ onto $H / H^{\prime}$. Since $H \in V$ and $H^{\prime} \leqq \Phi(H)$, the epimorphism $\lambda \alpha \theta: F \rightarrow H / H^{\prime}$ can be lifted to an epimorphism $\mu: F \rightarrow H$. Let $N=\operatorname{ker} \mu$. either

Now, it follows from results of Baer [1] (cf. Suzuki [12], p. 35) that

(a) $\phi_{1}: \mathscr{L}\left(G / G^{\prime}\right) \rightarrow \mathscr{L}\left(H / H^{\prime}\right)$ is induced by an isomorphism, or

(b) $G / G^{\prime}$ is finite and $k=2$.

In case (a), we may suppose that $\phi_{1}$ is induced by $\theta$, so that $\phi$ is compatible with the pair $\lambda, \mu$ on $F / F^{\prime}$. By theorem $2, \phi$ is compatible with the pair $\lambda, \mu$ on each factor group $F_{(i)} / F_{(i+1)}$. Thus

$$
\left(M \cap F_{(i)}\right) F_{(i+1)}=\left(N \cap F_{(i)}\right) F_{(i+1)} \quad(i=1,2, \cdots)
$$

and therefore, since $M<F_{(i)}, M=N$. Hence $G \cong H$. 
In case (b), $G$ itself is finite. Hence $G$ is the direct product of its Sylow subgroups $P_{1}, \cdots, P_{m}$. Suppose $P_{i}$ is a Sylow $p_{i}$-subgroup. Since $\phi$ is $\mathscr{N} \mathscr{L}$ and $P_{i}$ is noncyclic, $P_{i}^{\phi}$ is a $p_{i}$-group. It follows that $P_{1}^{\phi}, \cdots, P_{m}^{\phi}$ are the Sylow subgroups of $H$ (Suzuki [12], p. 5). If $P_{i}$ is abelian, it is the direct product of 2 cyclic groups of the same order and so $P_{i} \cong P_{i}^{\phi}$. It is therefore sufficient to prove the theorem when $G, H$ are finite (non-abelian) 2generator $p$-groups.

It is not difficult to see that, when $c=2$ or $3, G$ and $H$ are groups with generators $x, y$ and defining relations of the form

$$
\begin{array}{ll}
(c=2) & x^{p x}=y^{p x}=z^{p \phi}=u=v=1, \\
(c=3) & x^{p x}=y^{p x}=z^{p \phi}=u^{p v}=v^{p \phi}=1, \\
& {[x, u]=[y, u]=[x, v]=[y, v]=1,}
\end{array}
$$

where $z=[x, y], \quad u=[x, z], v=[y, z]$. Since corresponding factors $G_{(i)} / G_{(i+1)}, H_{(i)} / H_{(i+1)}$ are $\mathscr{N} \mathscr{L}$-isomorphic and so isomorphic, the indices $\alpha, \beta, \gamma, \delta$ are the same for both $G, H$. Hence $G \cong H$.

Suppose finally that $c \geqq 4$. We remark that $H / H_{(c)}$ is a homomorphic image of $F / F_{(c)}$, and that $H / H_{c}$ has the same order as $F / F_{c}$ (since $F / F_{(c)} \cong G / G_{(c)}$ and $G / G_{(c)}, H / H_{(c)}$ are $\mathcal{N} \mathscr{L}$-isomorphic $p$-groups). Therefore $H / H_{(c)} \cong F / F_{(c)}$ and so $N<F_{(c)}$. Set $K=G^{\prime} G^{p^{r}}, L=H^{\prime} H^{\nu^{r}}$, where $p^{r}$ is the exponent of $F_{(0)}$. Let $\phi^{\prime}: \mathscr{L}(G / K) \rightarrow \mathscr{L}(H / L)$ be the restriction of $\phi$ to $G / K$. Since $c \geqq 4$ and $F / F_{(c)} \cong G / G_{(c)} \cong H / H_{(c)}, G / G_{(4)}$ and $H / H_{(q)}$ are relatively free groups of class 3 and the exponent of $G_{(3)} / G_{(4)}$ is $p^{2} \geqq p^{r}$. By lemma 3, $\phi^{\prime}$ is induced by an isomorphism $\theta^{\prime}$. Thus, if we choose the isomorphism $\theta: G / G^{\prime} \rightarrow H / H^{\prime}$ of the first paragraph of the proof in such a way that $\theta^{\prime}$ is the restriction of $\theta$ to $G / K$, then $\phi$ is compatible with the pair $\lambda, \mu$ on $F / F^{\prime} F^{p^{r}}$. By theorem 2 (with each $P_{i} / Q_{i}$ equal to $F / F^{\prime} F^{p^{r}}$ ), $\phi$ is compatible with the pair $\lambda, \mu$ on $F_{(e)}$. Thus $\left(M \cap F_{(c)}\right) F_{(e+1)}=$ $\left(N \cap F_{(\mathrm{c})}\right) F_{(\mathrm{c}+1)}$, i.e. $M=N$. Hence $G \cong H$. This proves the theorem.

In some cases it is unnecessary to postulate that $H \in V$ since this follows from the existence of the $\mathscr{N} \mathscr{L}$-isomorphism $\phi$. The following is the simplest example.

COROLLARY 1. Let $V=V\left(p^{\mu}, c\right)$ be the variety of all nilpotent groups of class $c^{\prime} \leqq c$ and exponent $p^{\mu^{\prime}} \leqq p^{\mu}$. Let $G$ be a non-abelian almost free group of $V$ and $\phi$ an $\mathcal{N} \mathscr{L}$-isomorphism of $G$ onto a group $H$. Then $G \cong H$.

Recalling that every $\mathscr{L}$-isomorphism of a group of prime exponent is an $\mathscr{N} \mathscr{L}$-isomorphism, we get

COROLLARY 2. Let $G$ be a non-abelian, almost free group of $V(p, c)$ and $\phi$ an $\mathscr{L}$-isomorphism of $G$ onto a group $H$. Then $G \cong H$.

In particular: 
COROLlaRY 3. Let $G$ be a nilpotent group of class 2 and exponent $p$. Let $\phi$ be an $\mathscr{L}$-isomorphism of $G$ onto a group $H$. Then $G \cong H$.

Lie rings. The analogue of theorem 2 for Lie algebras over a principal ideal domain holds. (The arguments involving the mixed commutator preserving property can all be rephrased in terms of the commutator preserving property). Theorem 3 has the following analogue.

THEOREM 3'. Let $V$ be a nilpotent variety of Lie rings, $L$ a non-abelian almost free ring of $V$. Let $\phi$ be an $\mathscr{N} \mathscr{L}$-isomorphism of $L$ onto a member $M$ of $V$. Then $L \cong M$.

\section{References}

[1] Baer, R., The significance of the system of subgroups for the structure of the group, Amer. J. Math. 61 (1939), 1-44.

[2] Blackburn, N., On a special class of p-groups, Acta Math. 100 (1958), 45-92.

[3] Higman, G., Lie ring methods in the theory of finite nilpotent groups, Proc. Internat. Congress of Mathematicians, Edinburgh (1958), 307-312.

[4] Iwasawa, K., Uber die endlichen Gruppen und die Verbände ihrer Untergruppen, J. Univ. Tokyo 4-3 (1941), 171-199.

[5] Iwasawa, K., On the structure of infinite $M$-groups, Jap. J. Math. 18 (1943), 709-728.

[6] Lazard, M., Sur les groupes nilpotents et les anneaux de Lie, Ann. Sci. Ecole Norm. Sup. (3) 71 (1954), $101-190$.

[7] Pekelis, A. S., Structural isomorphisms of locally nilpotent torsion-free groups, Uspehi mat. nauk 18 (1963), pp. $187-190$ of part 3(iii). (Russian).

[8] Pekelis, A. S. and Sadovskii, L. E., Projections of a metabelian torsion-free group, Doklady Akad. Nauk S.S.S.R. 151 (1963), 42-44 (English translation in Soviet Math. 4 (1963), 918-920).

[9] Rottländer, A., Nachweis der Existenz nicht-isomorpher Gruppen von gleicher Situation der Untergruppen, Math. Z. 28 (1928), $641-653$.

[10] Smel'kin, Free polynilpotent groups, Doklady Akad. Nank S.S.S.R. 151 (1963), $73-75$ (English translation in Soviet Matb. 4 (1963), 950-953).

[11] Spring, R. F., Lattice isomorphisms of funite non-abelian groups of exponent $p$, Proc. Amer. Math. Soc. 14 (1963), $407-413$.

[12] Suzuki, M., Structure of a Group and the Structure of its Lattice of Subgroups (Springer, Berlin, 1956).

[13] Wiman, A., Uber mit Diedergruppen verwandte p-Gruppen, Arkiv för Mat., Astron. och Fys. 33A (1946).

Mathematics Department, University of Sydney. 